\title{
An accurate automated blood pressure device for use in pregnancy and pre-eclampsia: the Microlife 3BTO-A
}

\author{
A. Reinders, A.C. Cuckson, J.T.M. Lee, A.H. Shennan
}

Objective To assess the accuracy of an automated blood pressure device (Microlife 3BTO-A) in pregnancy and pre-eclampsia according to the British Hypertension Society (BHS) protocol.

Design Prospective observational study.

Setting Antenatal ward and clinics at Guy's and St Thomas' Hospital, London, UK.

Population One hundred and five pregnant women including 35 women with non-proteinuric hypertension and 35 with pre-eclampsia.

Methods Two trained observers took nine sequential same-arm measurements from each woman. Measurements alternated between a mercury sphygmomanometer and the device.

Main outcome measures Grading criteria of the BHS protocol (A/B grade $=$ pass; C/D grade $=$ fail).

Results The device passed the BHS protocol by achieving an A/B grade. It also achieved criteria of the Association for the Advancement of Medical Instrumentation for systolic and diastolic pressures respectively, in normotensive $[-0.5(5.7) \mathrm{mmHg} ;-0.07(7.7) \mathrm{mmHg}]$, non-proteinuric hypertensive $[-3.3(6.9) \mathrm{mmHg} ;-2.4(6.6) \mathrm{mmHg}]$ and pre-eclamptic pregnancy $[-4.1(6.4) \mathrm{mmHg} ;-1.3(7.9) \mathrm{mmHg}]$.

Conclusion The Microlife 3BTO-A can be recommended for use in a pregnant population, including preeclampsia, according to the BHS protocol.

\section{INTRODUCTION}

Intracranial haemorrhage is the leading cause of maternal mortality in women with pre-eclampsia. ${ }^{1}$ Severe hypertension is likely to be the most significant aetiological factor related to this cause and effective anti-hypertensive treatment is crucial. It is not known if inaccurate blood pressure measurement contributes to maternal mortality, but it is well recognised that automated devices systematically underestimate both systolic and diastolic blood pressure in preeclampsia and often by clinically significant amounts.

The 'gold standard' (mercury sphygmomanometer) has been criticised for its associated observer errors and environmental safety groups have concerns about mercury toxicity. Over the past 20 years various mercury-independent alternatives (aneroid and automated) have been introduced, the majority of which do not use auscultation to determine blood pressure. It is imperative that these devices be assessed according to recognised protocols to determine their accuracy compared with the mercury sphygmomanometer.

The Association for the Advancement of Medical Instrumentation (AAMI), ${ }^{2}$ the British Hypertension Society

Maternal and Fetal Research Unit, St Thomas' Hospital, London, $U K$

Correspondence: Professor A. H. Shennan, Maternal and Fetal Research Unit, 10th floor North Wing, St Thomas' Hospital, Lambeth Palace Road, London SE1 7EH, UK.

(C) RCOG 2005 BJOG: an International Journal of Obstetrics and Gynaecology
$(\mathrm{BHS})^{3}$ and most recently the European Society for Hypertension $(\mathrm{ESH})^{4}$ have all published protocols with a view to standardise both the method of assessment and the level of accuracy required of a device in order to be recommended for clinical use.

Only one automated device, the Omron MIT, ${ }^{5}$ has demonstrated sufficient accuracy to be appropriate for clinical use in pre-eclampsia and there is an urgent need for more accurate devices. Of the eight other devices ${ }^{6-11}$ - deemed accurate in adults - that were evaluated in hypertensive pregnancy, the vast majority under-read in pre-eclampsia by clinically significant amounts (i.e. by a mean of more than $5 \mathrm{mmHg}$ ). It is therefore important for devices intended for use in an obstetric population to be assessed in this group specifically.

The Microlife 3BTO-A (Microlife, Taipei, Taiwan) is a compact device suitable for self-measurement. The device previously achieved the highest possible grade for accuracy (A/A) in an adult population ${ }^{12}$ according to the BHS protocol. In this study we evaluated its accuracy in a pregnant population, including women with non-proteinuric hypertension and pre-eclampsia.

\section{METHODS}

The study was performed by two observers, trained in blood pressure measurement (BHS specifications). Women were recruited from the antenatal ward and clinics at two large teaching hospitals in London, UK (Guy's and 
Table 1. BHS grading criteria.

\begin{tabular}{llcr}
\hline Grade & \multicolumn{2}{c}{$\begin{array}{c}\text { Absolute difference between standard and } \\
\text { test device }(\mathbf{m m H g})\end{array}$} \\
\cline { 2 - 4 } & $\leq 5$ & $\leq 10$ & $\leq 15$ \\
\hline Cumulative percentage of readings (\%) & & \\
A & 60 & 85 & 95 \\
B & 50 & 75 & 90 \\
C & 40 & 65 & 85 \\
D & & Worse than C & \\
\hline
\end{tabular}

St Thomas' Hospitals). Ethical approval was obtained and participants were asked to give written informed consent.

One hundred and five women were recruited to the study, including 35 women with non-proteinuric hypertension and 35 women with pre-eclampsia. Only women over the age of 18 years and with a gestation greater than 22 weeks were approached to take part in the study. Women with any arrhythmia or those in which Korotkoff sounds did not disappear, or with a diastolic $<40 \mathrm{mmHg}$, were excluded. Korotkoff 5 was used to identify the diastolic pressure.

Pre-eclampsia was defined as a diastolic blood pressure of $\geq 90 \mathrm{mmHg}$ on two separate occasions more than 4 hours apart or a single reading $>110 \mathrm{mmHg}$ accompanied by proteinuria of $>0.3 \mathrm{~g}$ on a 24-hour sample or $2+$ on reagent strip. ${ }^{13}$ Non-proteinuric hypertension was defined as those women who fulfilled only the hypertension criteria of the above definition.

The study was performed according to guidelines of the 1993 BHS protocol. A calibration check was performed according to guidelines in the protocol. The device underwent a minimum of 400 inflations in its intended environment and calibration was rechecked. This part of the protocol was undertaken during the assessment of the same device in an adult population ${ }^{12}$ directly preceding this study and therefore not repeated. Calibration was rechecked before the start of this study and the device achieved targets as outlined in the protocol.
Demographic information such as age, height and gestation was obtained from each patient. Blood pressure measurements were taken in a quiet room with the subject seated and the arm supported at heart level. Arm circumference was measured at the approximate midpoint of the upper arm to determine the appropriate cuff size to be used. Two cuff sizes were available: normal adult $(22-32 \mathrm{~cm})$ and large adult $(32-42 \mathrm{~cm})$. The device was connected to a laptop computer with recording software (LabView) to facilitate additional ongoing analysis not described in this paper.

Nine sequential same arm measurements were taken alternating between the reference (mercury sphygmomanometer) and the test device (Microlife 3BTO-A). Auscultatory readings were taken using an electronic stethoscope (Welch Allyn sensor-based stethoscope model 5079-400) and a distributor box enabled the second observer to listen to Korotkoff sounds. More than 30 seconds but less than 1 minute was allowed between readings to reduce the effect of venous congestion and to limit variability. The patient was advised to relax, avoid talking and to keep the arm as still as possible as the device measurements could be influenced by movement. The patient was also asked to advise us of any discomfort during the procedure.

Of the nine measurements taken, only the last seven were used in analysis. The first manual reading was used to classify the subject in the appropriate category as specified in the protocol and the first device reading was used to 'orientate' the device to the patient. The mean differences and standard deviation between device and observer was calculated and device was graded according to criteria of the BHS protocol (Table 1). The device should achieve percentages greater than or equal to those in the table to achieve a particular grade. Furthermore, a visual representation of the accuracy of the device is provided using Bland-Altman plots. ${ }^{14}$ This has the benefit of establishing at first glance whether there are any trends (e.g. increased error at increased pressures) or cases of extreme inaccuracy.

Table 2. Grading, cumulative percentage of differences between mercury sphygmomanometer and test device that varied by $\leq 5, \leq 10$ and $\leq 15 \mathrm{mmHg}$, mean pressure and mean difference of the pressure between observer and test device.

\begin{tabular}{|c|c|c|c|c|c|c|}
\hline & \multirow[t]{2}{*}{ Grade } & \multicolumn{3}{|c|}{$\begin{array}{c}\text { Difference between standard and test } \\
\text { device }(\mathrm{mmHg})\end{array}$} & \multirow[t]{2}{*}{$\begin{array}{l}\text { Mean [SD] } \\
(\mathbf{m m H g})\end{array}$} & \multirow[t]{2}{*}{$\begin{array}{c}\text { Mean }[\mathrm{SD}] \text { of } \\
\text { differences }(\mathrm{mmHg})\end{array}$} \\
\hline & & $\leq 5$ & $\leq 10$ & $\leq 15$ & & \\
\hline \multicolumn{7}{|c|}{ Adult population $(n \equiv 255)^{*}$} \\
\hline SBP & A & 64 & 87 & 96 & $134.9[28.1]$ & $-1.7[7.4]$ \\
\hline DBP & A & 68 & 89 & 97 & 84 [19.7] & $-2.1[6.3]$ \\
\hline SBP & A & 63 & 87 & 97 & $125[17.5]$ & $-2.7[6.3]$ \\
\hline DBP & $\mathrm{B}$ & 57 & 83 & 97 & $79.2[12.6]$ & $-1.3[7.4]$ \\
\hline
\end{tabular}

$\mathrm{SBP}=$ systolic blood pressure; $\mathrm{DBP}=$ diastolic blood pressure; $\mathrm{SD}=$ standard deviation.

* Previously published data in non-pregnant adults. ${ }^{17}$

** Including hypertensive pregnancy. 
Table 3. Grading, cumulative percentage of differences between mercury sphygmomanometer and test device that varied by $\leq 5$, $\leq 10$ and $\leq 15 \mathrm{mmHg}$, mean pressure and mean difference of the pressure between observer and test device for normotensive and hypertensive pregnancy.

\begin{tabular}{|c|c|c|c|c|c|c|}
\hline & \multirow[t]{2}{*}{ Grade } & \multicolumn{3}{|c|}{$\begin{array}{c}\text { Difference between standard and test } \\
\text { device }(\mathrm{mmHg})\end{array}$} & \multirow[t]{2}{*}{$\begin{array}{l}\text { Mean [SD] } \\
(\mathbf{m m H g})\end{array}$} & \multirow[t]{2}{*}{$\begin{array}{c}\text { Mean }[\mathrm{SD}] \text { of } \\
\text { differences }(\mathrm{mmHg})\end{array}$} \\
\hline & & $\leq 5$ & $\leq 10$ & $\leq 15$ & & \\
\hline \multicolumn{7}{|c|}{ Normotensive $(n=105)$} \\
\hline SBP & A & 69 & 95 & 99 & $108[11]$ & $-0.5[5.7]$ \\
\hline DBP & B & 55 & 82 & 97 & $66[12]$ & $-0.07[7.7]$ \\
\hline SBP & B & 60 & 80 & 95 & $132[16]$ & $-3.3[6.9]$ \\
\hline DBP & B & 58 & 85 & 100 & 85 [12] & $-2.4[6.6]$ \\
\hline \multicolumn{7}{|c|}{ Pre-eclampsia $(n=105)$} \\
\hline SBP & A & 62 & 86 & 96 & 137 [26] & $-4.1[6.4]$ \\
\hline DBP & B & 59 & 82 & 93 & $87[14]$ & $-1.3[7.9]$ \\
\hline
\end{tabular}

$\mathrm{SD}=$ standard deviation

\section{RESULTS}

The Microlife 3BTO-A achieved an overall grade A for systolic pressures and grade B for diastolic pressures (Table 2). Results for normotensive, hypertensive and pre-eclamptic pregnancies are shown in Table 3. AAMI criteria (mean $<5 \mathrm{mmHg}[<8]$ ) were met overall and in all groups individually.

Bland-Altman plots ${ }^{14}$ show the difference in blood pressure between the test device and the better observer plotted against the mean pressure of the device and the observer.
Results are shown for normotensive, non-proteinuric hypertensive and pre-eclamptic pregnancy. Figures 1 and 2 indicate the results for systolic and diastolic pressures, respectively.

Demographic information is displayed in Table 4. There were no statistically significant differences between the groups $(P>0.1)$. Fourteen pre-eclamptic women and six women who had non-proteinuric hypertension had an arm circumference greater than $32 \mathrm{~cm}$. All the women in the normotensive group had an arm circumference $<32 \mathrm{~cm}$. The range of proteinuria (24-hour collection) was $0.3-15.52 \mathrm{~g} / \mathrm{dL}$ for women with pre-eclampsia. Thirteen

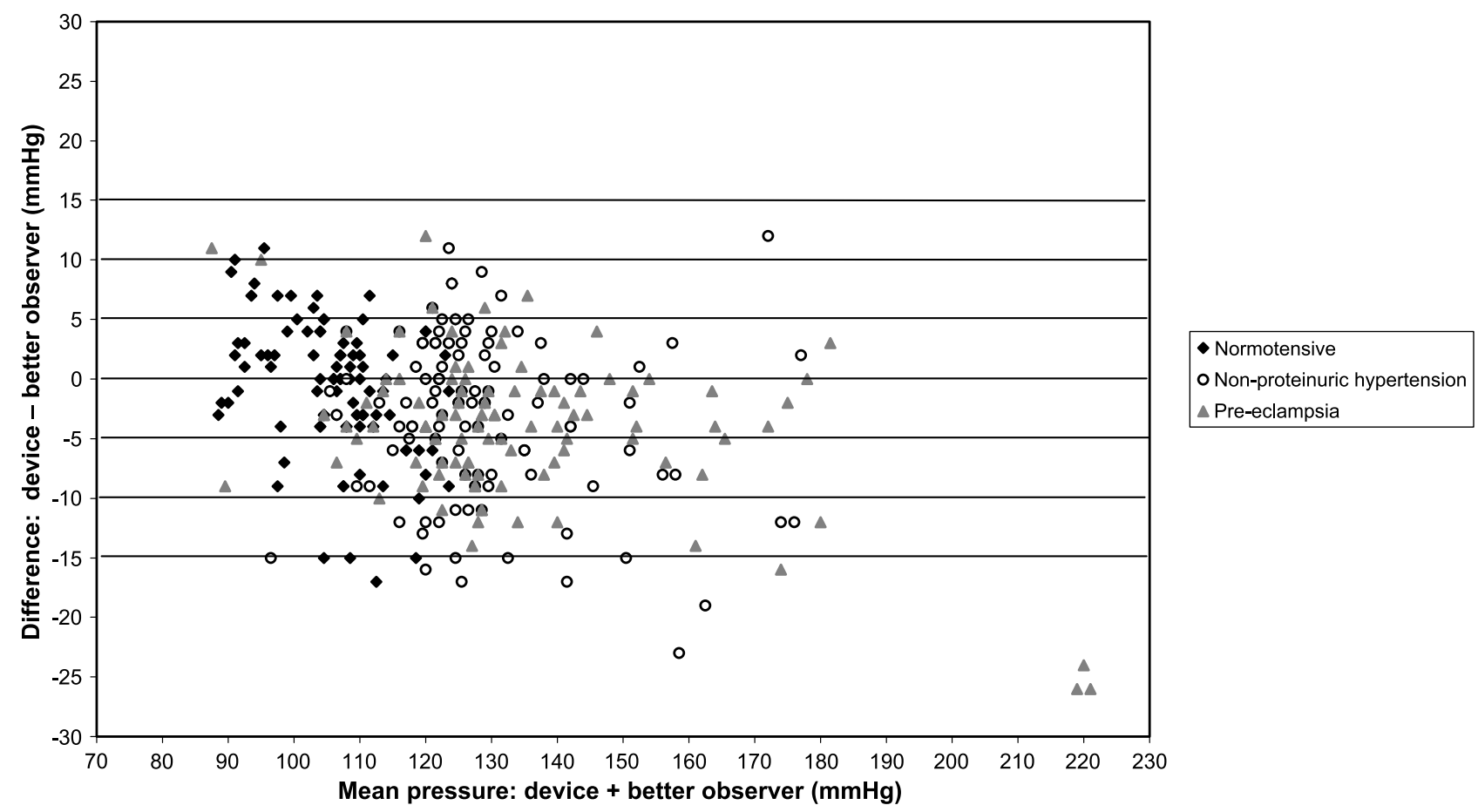

Fig. 1. Plot of the pressure difference between the better observer and the test device and the mean pressure of that observer and the device for systolic pressure, in normotensive and hypertensive pregnancy.

(C) RCOG 2005 BJOG: an International Journal of Obstetrics and Gynaecology 112, pp. 1-6 


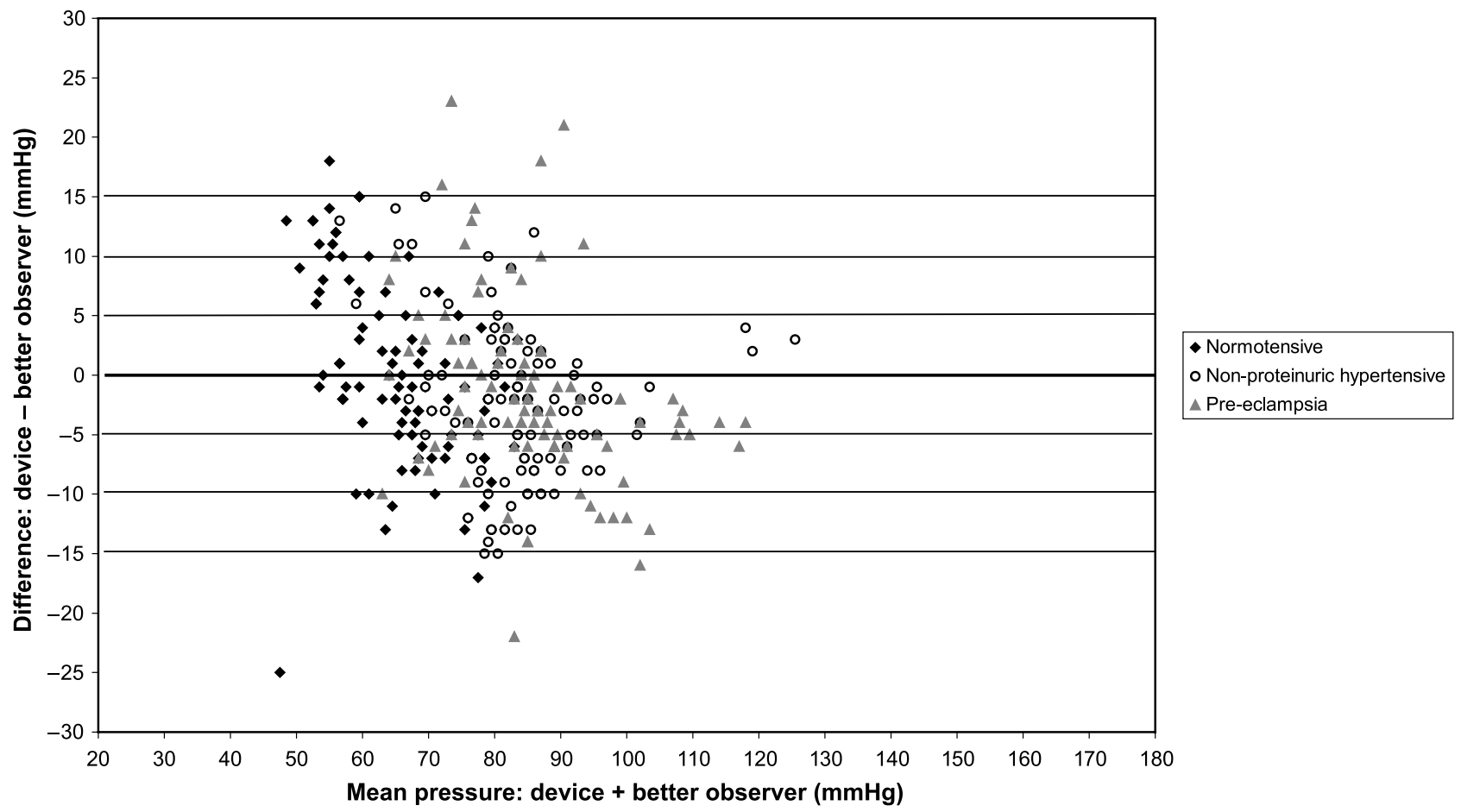

Fig. 2. Plot of the pressure difference between the better observer and the test device and the mean pressure of that observer and the device for diastolic pressure in normotensive and hypertensive pregnancy.

women with non-proteinuric hypertension and 20 women with pre-eclampsia were receiving anti-hypertensive treatment at the time of recruitment.

\section{COMMENT}

The Microlife 3BTO-A can be used clinically for BP assessment in pregnancy and is one of only two devices recommended in pre-eclampsia.

Various devices, recommended in an adult and pregnant population according to the BHS criteria, under-estimated quite significantly when assessed in pre-eclampsia. Mean differences reported have been as great as $15 \mathrm{mmHg}$ when compared with mercury sphygmomanometry ${ }^{8}$ and $25 \mathrm{mmHg}$ when compared with intra-arterial measurements ${ }^{7}$ and individual readings have been far greater.

To our knowledge only one device, the Omron MIT (self-measurement), has achieved the BHS criteria in preeclampsia. ${ }^{5}$ This device uses inflationary oscillometry (i.e. it determines the blood pressure while the cuff is inflating and then rapidly deflates). This is contrary to most oscillometric devices, which measure blood pressure during deflation of the cuff. As pre-eclampsia is associated with decreased arterial compliance and an increase in interstitial tissue oedema, it was thought that transmission of the pressure wave could be delayed using a deflation method (thereby under-estimating true blood pressure). Using an inflationary method would allow immediate detection of the signal and therefore be more accurate.
Our study using the Microlife 3BTO-A has reconfirmed that a good algorithm is vital (using a deflationary method), but as no other inflationary device has been assessed according to a recognised protocol, it is uncertain whether the accuracy of the Omron MIT can be attributed to inflationary oscillometry or simply a good algorithm (or a combination of both).

It is well known that automated devices tend to show increased error at higher pressures. In the assessment of the Microlife 3BTO-A, the device shows greater error in preeclampsia compared with normotensive pregnancy [SBP -4.1 (6.4) vs -0.5 (5.7); DBP -1.3 (7.9) vs -0.1 (7.7)]. Whether using inflationary methods could possibly rectify or reduce this error needs further investigation.

Another factor contributing to device accuracy is the use of an appropriately sized cuff. Pre-eclampsia is associated

Table 4. Demographic information. Values are presented as mean [SD].

\begin{tabular}{lccc}
\hline & Normotensive & $\begin{array}{c}\text { Non-proteinuric } \\
\text { hypertensive }\end{array}$ & Pre-eclampsia \\
\hline Age (years) & $31[5.6]$ & $33[5.3]$ & $33[5.7]$ \\
CI (age) & $28.6-32.4$ & $30.9-34.6$ & $31.3-35.2$ \\
Gestation (weeks) & $32[5.1]$ & $35[4.6]$ & $35[4.7]$ \\
CI (gestation) & $31-34$ & $33-36$ & $33-36$ \\
Second trimester & 8 & 3 & 6 \\
Third trimester & 27 & 32 & 29 \\
Primips & 16 & 22 & 18 \\
\hline
\end{tabular}

$\mathrm{CI}=95 \%$ confidence intervals. 
with oedema due to increased interstitial permeability and it is thought that women with a BMI $>30$ are at an increased risk for pre-eclampsia. In this study almost half of all pre-eclamptic women and a fifth of women with non-proteinuric hypertension had an arm circumference $>32 \mathrm{~cm}$.

Devices intended for self-measurement, like the Microlife 3BTO-A, can also be used by patients to measure their blood pressure in their home environment. It reduces/ eliminates the white coat effect and could impact on the cost and effort involved for women who have to come to hospital just to have their blood pressure checked. Results from studies done in both a non-pregnant population ${ }^{15}$ and a pregnant population ${ }^{16}$ have been encouraging. Patient compliance and reporting of device measurements are enhanced as most of these devices now either have a memory facility or the capability to connect to a PC or printer to obtain a printout of readings.

Home monitoring by the patient using new technologies seems feasible and beneficial to both the patient and the clinician. Waugh et al. ${ }^{17}$ did a small study investigating the use of home blood pressure monitoring in combination with urinalysis. Results were very positive and low risk as well as high risk populations are currently being assessed to determine the application of this point-of-care technology.

Only one robust device is currently recommended for use in pregnancy, although it did not achieve BHS criteria when assessed in pre-eclampsia. ${ }^{9}$ As we have no robust device suitable for use in the clinical setting (with regard to pre-eclampsia) and there is pressure to phase out the mercury sphygmomanometer, would it be feasible to use a device intended for self-measurement?

We are only aware of one study in which a selfmeasurement device was assessed in the clinical setting. Lo et al. ${ }^{18}$ used the Omron HEM 705-CP to compare blood pressure readings to that obtained by mercury sphygmomanometry in pre-eclamptic women on the antenatal ward. The methodology of the study was ad hoc and the Omron device used failed BHS criteria when assessed in preeclampsia in a previous study. ${ }^{5}$

If self-measurement devices are to be used in the clinical setting, then consideration should be given to issues like accuracy, lifespan, cost and monitoring facilities.

Devices intended for use in a pregnant population should be assessed according to a recognised protocol in a pregnant population and accuracy should not be assumed from a validation conducted in an adult population. Currently, only the BHS protocol and AAMI make provision for the assessment of a blood pressure measuring device in pregnancy. However, it does not make specific provision for women with pre-eclampsia.

Furthermore, the lifespan of any blood pressure measuring device greatly depends on the capacitive sensor. This sensor consists of two round copper plates that press against each other with pressure changes in the cuff. These pressures are translated to a digital chip and then converted to a digital signal. The lifespan of these sensors can vary from 10,000 to 30,000 measurements. The longer the lifespan, the more expensive the sensor. Most devices for selfmeasurement are therefore likely to have a capacitive sensor with a shorter lifespan in order to keep the cost down, whereas robust devices are likely to have a more expensive sensor with a longer lifespan. However, robust devices are often capable of more intensive monitoring facilities (i.e. temperature, saturation, etc.) and these parameters will also influence the lifespan and cost of the device.

As no other robust device can currently be recommended for use in pre-eclampsia, it might be feasible to use a selfmeasurement device, depending on the level of monitoring required and the frequency of use. The Microlife 3BTO-A can be recommended for use in pregnancy, including those women who have non-proteinuric hypertension and preeclampsia. The role of self-measurement devices in the clinical setting warrants further investigation.

\section{Acknowledgments}

The authors would like to thank staff and patients at Guy's and St Thomas' Hospitals for their help. Our thanks also extend to the Charitable Foundation of Guy's and St Thomas' Hospital for funding this study and to Microlife for providing financial support for previous validation work.

\section{References}

1. Why mothers die 1997-1999. The fifth report of the Confidential Enquiries into Maternal Deaths in the United Kingdom. Available: http:// www.cemd.org.uk.

2. Association for the Advancement of Medical Instrumentation. American National Standard for Electronic or Automated Sphygmomanometers ANSI/AAMI SP10-1992. Arlington, VA: AAMI, 1993.

3. O'Brien E, Petrie J, Littler W, et al. The British Hypertension Society protocol for the evaluation of blood pressure measuring devices. J Hypertens 1993;11(Suppl 2):S43-S62.

4. O'Brien E, Pickering T, Asmar R, et al. Working Group on Blood Pressure Monitoring of the European Society of Hypertension International Protocol for validation of blood pressure measuring devices in adults. Blood Press Monit 2002;7:3-17.

5. Golara M, Benedict A, Jones C, Randhawa M, Poston L, Shennan AH. Inflationary oscillometry provides accurate measurement of blood pressure in pre-eclampsia. Br J Obstet Gynaecol 2002;109(10):11431147 (October).

6. Gupta M, Shennan A, Halligan A, Taylor DJ, de Swiet M. Accuracy of oscillometric blood pressure monitoring in pregnancy and preeclampsia. Br J Obstet Gynaecol 1997;104:350-355.

7. Natarajan P, Shennan AH, Penny J, Halligan AW, de Swiet M, Anthony J. Comparison of auscultatory and oscillometric automated blood pressure monitors in the setting of pre-eclampsia. Am J Obstet Gynecol 1999;181:1203-1210.

8. Quinn M. Automated blood pressure measurement devices: a potential source of morbidity and pre-eclampsia. Am J Obstet Gynecol 1994; 170(5): $1303-1307$.

9. Reinders A, Cuckson AC, Jones CR, Poet R, O'Sullivan G, Shennan AH. Validation of the Welch Allyn 'Vital Signs' blood pressure measurement 
device in pregnancy and pre-eclampsia. Br J Obstet Gynaecol 2003;110: $134-138$.

10. Armstrong CA, Shennan AH, Rushbrook J, Hussain W, Halligan AW. Validation of the Omron Rx wrist blood pressure monitor for use in pregnancy. In press.

11. Franx A, Van der Post JAM, van Montfrans GA, Bruinse HW. Comparison of an osculatory versus an oscillometric ambulatory blood pressure monitor in normotensive, hypertensive and pre-eclamptic pregnancy. Hypertens Pregnancy 1997;16:187-202.

12. Cuckson AC, Reinders A, Shabeeh H, Shennan AH. Validation of the Microlife 3BTO-A oscillometric blood pressure monitoring device according to a modified British Hypertension Society protocol. Blood Press Monit 2002;7:319-324.

13. Davey DA, MacGillivray I. The classification and definition of the hypertensive disorders of pregnancy. Am J Obstet Gynecol 1988; 158(4):892-898.

14. Bland JM, Altman DG. Statistical methods for assessing agreement between two methods of clinical measurement. Lancet 1986;328: 307-310.

15. Chatellier G, Dutrey-Dupagne C, Vaur L, et al. Home self blood pressure measurement in general practice (the SMART study). Am J Hypertens 1996;9:644-652.

16. Ross McGill H, Hewison J, Hirst J, et al. Antenatal home blood pressure monitoring: a pilot randomised controlled trial. Br J Obstet Gynaecol 2000;107:217-221.

17. Waugh J, Bosio P, Shennan A, Halligan A. Inpatient monitoring on an outpatient basis: managing hypertensive pregnancies in the community using automated technologies. J Soc Gynecol Investig 2001;8: $14-17$.

18. Lo C, Taylor RS, Gamble G, McCowan L, North RA. Use of automated home blood pressure monitoring in pregnancy: is it safe? Am J Obstet Gynecol 2002;187:1321-1328.

Accepted 2 December 2004 